\title{
TRANSVERSE BEAM FEEDBACK SYSTEM IN THE PLS
}

\author{
J. Y. Huang, M. K. Park, M. Kwon, M. Yoon and I. S. Ko \\ Pohang Accelerator Laboratory, POSTECH \\ Pohang 790-784, Korea
}

\begin{abstract}
A bunch by bunch transverse feedback system, as a complement of the longitudinal feedback system required for the suppression of the coupled bunch beam instabilities, has been developed and undergoing testing in the PLS. Major components are two beam oscillation detectors, betatron phase adjuster, notch filters and a stripline kicker. Each system component and its functions are described with simple trigonometric calculations. Preliminary result of the beam test has shown $30 \mathrm{~dB}$ damping of the beam oscillation.
\end{abstract}

\section{INTRODUCTION}

The PLS is a $2 \mathrm{GeV}$ electron synchrotron light source dedicated to the beamline users since 1995. During the operation, various kind of beam instabilities have been observed. In particular, low frequency beam oscillation around $100 \mathrm{~Hz}$ and the longitudinal coupled bunch instability induced by higher order modes of $\mathrm{rf}$ cavities (HOM) are typical [1]. For the suppression of these instabilities, a PEP-II type longitudinal feedback system [2] will be developed.

Although the transverse coupled bunch instability has not caused any problem in PLS, inserting of the narrow gap chambers for the planned insertion devices will generate large transverse resistive wall impedance, and, consequently, the spontaneous transverse coupled bunch instability will be possible. It is also well experienced in ALS that a transverse feedback system is required as a complement for the longitudinal feedback system to suppress the coupled bunch instabilities properly [3].

A PLS transverse feedback system is developed and is undergoing testing now. It operates as a bunch by bunch feedback system in time domain [3][4]. Frequency domain bandwidth of the system is $250 \mathrm{MHz}$, which is the highest coupled bunch oscillation frequency observable from $500 \mathrm{MHz}$ bunch train. The PLS transverse feedback system consists of pickup electrodes, signal processing electronics, power amplifiers, and a stripline kicker. Fig. 1 shows an overview of the transverse feedback system. The first beam study has shown $30 \mathrm{~dB}$ damping of the beam oscillation in full bandwidth. In this paper, we describe the design and the preliminary test result of the transverse feedback system.

\section{FEEDBACK SYSTEM}

Two fast beam oscillation detectors are used for the control of $\pi / 2$ phase relation between the pickup signal and the feedback kicker signal. Signal detection is done at the third harmonic of $500 \mathrm{MHz}$ rf frequency $(1.5 \mathrm{GHz})$. A notch filter made with two coaxial delay lines is used to suppress the DC beam signal [4]. Overall bandwidth of the system is $100 \mathrm{kHz}$ to $250 \mathrm{MHz}$. Four 100 watt power amplifiers are used to drive the feedback kicker providing $65 \mathrm{~dB}$ total system gain. Each system component and its functions are described with simple trigonometric calculations and the test results are discussed in the following sections.

\subsection{Pickup Electrodes}

Two orbit BPMs, 6PM6 and 7PM6, are selected as the pickup electrodes, which have been reserved for beam diagnostics. They are located at the finite dispersion function region and 70 degrees apart in the betatron phase. High frequency capacitance of a pickup electrode is $2 \mathrm{pF}$, and the rise-fall time of the pickup signal is much shorter than 2 ns bunch seperation. RF response of a pickup electrode is also clean without any resonant spectral structure up to $12 \mathrm{GHz}$ [5]. With two selected BPMs, $\pi / 2$ betatron phase relation between the pickup electrode and the kicker can be controlled by adjusting attenuators $a_{1}$ and $a_{2}$.

When two pickup electrodes are $\varphi_{0}$ apart in betatron phase, beam signals $x_{1}$ and $x_{2}$ can be written as

$$
\begin{aligned}
& x_{1}=a_{1} \sqrt{\beta_{1}} \sin \left(\varphi_{I}(s)\right), \\
& x_{2}=a_{2} \sqrt{\beta_{2}} \sin \left(\varphi_{I}(s)+\varphi_{0}\right),
\end{aligned}
$$

and the sum signal $x$ is

$$
\begin{aligned}
x= & x_{1}+x_{2} \\
= & {\left[\left(a_{1} \sqrt{\beta_{1}}+a_{2} \sqrt{\beta_{2}} \cos \varphi_{0}\right)^{2}+a_{2}^{2} \beta_{2} \sin ^{2} \varphi_{0}\right]^{1 / 2} } \\
& x \sin \left(\varphi_{1}(s)+\Delta \varphi\right)
\end{aligned}
$$

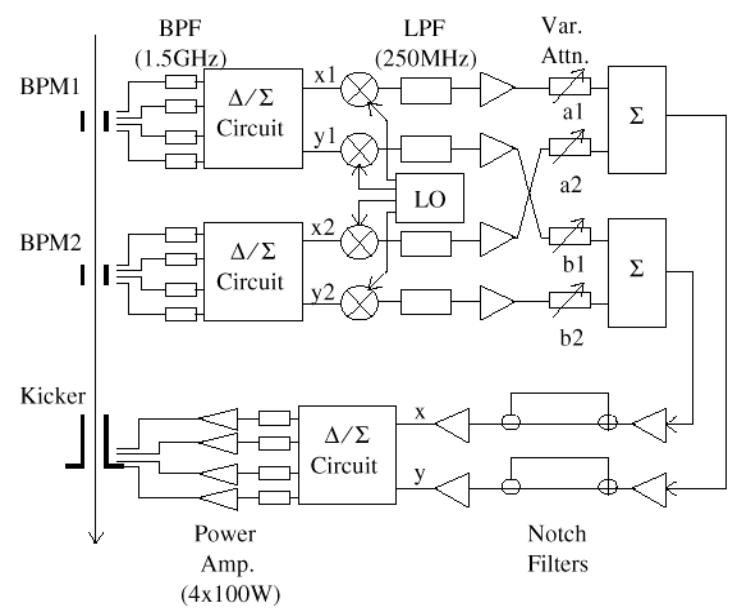

Figure 1: An overview of the transverse feedback system 
where the phase change $\Delta \varphi$ is

$$
\Delta \varphi=\tan ^{-1}\left[a_{2} \sqrt{\beta_{2}} \sin \varphi_{0} /\left(a_{1} \sqrt{\beta_{1}}+a_{2} \sqrt{\beta_{2}} \cos \varphi_{0}\right)\right]
$$

By adjusting $a_{1}$ and $a_{2}$, we can control $\Delta \varphi$ as much as $2 \pi$. Since we select BPMs located at the same betatron functions, where $\beta_{h}=2.1326$ and $\beta_{v}=3.3119$, the betatron phase delay is controlled only by the ratio of the attenuations $a_{2} / a_{1}$.

\subsection{Signal Processing Electronics}

Pickup signals are delivered to the frontend electronics via phase matched coaxial cables. Since the beam signal is largest at the third harmonic $(-10 \mathrm{dBm}$ at $100 \mathrm{~mA})$ of $500 \mathrm{MHz}$ rf frequency, $1.5 \mathrm{GHz}$ band is selected for the detection of beam oscillation. The rf detection electronics consists of $1.5 \mathrm{GHz} \pm 300 \mathrm{MHz}$ band pass filters, rf hybrids, mixers, $1.5 \mathrm{GHz}$ local oscillator, and the 250 $\mathrm{MHz}$ low pass filter at the mixer output. Detected signals of BPM1 and BPM2 are combined in the power divider with attenuation coefficients $a_{1}$ and $a_{2}$, which are calculated from Eq.(4). Four attenuators $a_{1}, a_{2}, b_{1}, b_{2}$ are computer controlled to accommodate the change of operation tunes. DC component of the beam signal, which appears as the revolution harmonics of the beam spectrum, is suppressed by two correlator notch filters. A notch filter is made with two coaxial delay lines which differ in delay time by the ring revolution period $T$. Output signal $x(t)$ can be written as the difference of two input signals with attenuations $\alpha$ and $\beta$ as

$$
x(t)=\alpha x_{0}(t)-\beta x_{0}(t-T) .
$$

Since the notch depth is sensitive to the mismatch of the delays and attenuations of two delay lines, they are precisely adjusted by a variable delay line and a variable attenuator to obtain the best notch depthes. To see how the notch frequency and the notch depth is sensitive to the delay error $\delta T$ and the mismatch of attenuation $\alpha$ and $\beta$ of delay lines, we consider the frequency domain spectrum:

$$
x(\omega) \propto\left[\alpha^{2}+\beta^{2}-2 \alpha \beta \cos (\omega T+\omega \delta T)\right]^{1 / 2} .
$$

The notch frequency shift is $\delta f=\omega \delta T / 2 \pi T=f_{\text {rev }} \delta T / T$ and the notch depth $R$, defined as $x_{\max } / x_{\text {min }}$, is

$$
R=(\alpha+\beta) /(\alpha-\beta) \text {. }
$$

When $\beta / \alpha$ is adjusted within $1 \%$, the notch depth is $46 \mathrm{~dB}$.

Phase change by the notch filter should also be considered in tuning the phase delay between pickup and kicker of the feedback system. From Eq. (5),

$$
\begin{aligned}
x(t) & =\left[\left(\alpha-\beta \cos ^{2}\left(2 \pi v_{x}\right)+\beta^{2} \sin ^{2}\left(2 \pi v_{x}\right)\right]^{1 / 2}\right. \\
& x \sin (\omega t+\Delta \varphi),
\end{aligned}
$$

where the phase change $\Delta \varphi$ is

$$
\Delta \varphi=\tan ^{-1}\left[\beta \sin \left(2 \pi v_{x}\right) /\left(\alpha-\beta \cos \left(2 \pi v_{x}\right)\right)\right] .
$$

In practice, we can set $\alpha=\beta=1$, and the phase shift by the notch filter is

$$
\Delta \varphi=\tan ^{-1}\left[\cot \left(2 \pi v_{x}\right)\right]=\pi / 2-\pi v_{x} .
$$

For the PLS system, $v_{x}=14.28$ and $v_{y}=8.18$, and consequently $\Delta \varphi_{x}=0.22 \pi, \Delta \varphi_{y}=0.32 \pi$.

\subsection{Power Amplifier}

Beam induced transverse kick voltage $V_{\perp}$ by the transverse impedance $Z_{\perp}$ is given by

$$
V_{\perp}=I_{0} \delta x Z_{\perp}
$$

where $\delta x$ is amplitude of the transverse oscillation and $I_{0}$ is the beam current. The required power of the kicker should provide more power than the beam induced power $P_{b}$ to damp the oscillation:

$$
P_{b}=V_{\perp}^{2} / 2 R_{k}
$$

where $R_{k}$ is the kicker shunt impedance.

For the PLS, the transverse HOM impedance $Z_{\perp}$ of the cavity is practically less than $10 \mathrm{M} \Omega$ and the shunt impedance $R_{k}$ of the transverse feedback kicker is $6.5 \mathrm{k} \Omega$ at $250 \mathrm{MHz}$. When $I_{0}=100 \mathrm{~mA}$ and $\delta x=1 \mathrm{~mm}, V_{\perp}=1$ $\mathrm{kV}$ and $P_{b}=77$ watt respectively. We need minimum 310 watt power amplifier when the beam current reaches 400 $\mathrm{mA}$. Four 100 watt, $50 \mathrm{~dB}$ gain power amplifiers are purchased for the PLS transverse feedback system. The 3 $\mathrm{dB}$ bandwidth of the amplifier is $10 \mathrm{kHz}$ to $230 \mathrm{MHz}$.

\subsection{Kicker}

A four-stripline type kicker is used for both horizontal and vertical kick instead of the seperate horizontal and vertical electrodes because of the lack of free space in the storage ring. Striplines are $17 \mathrm{~mm}$ wide and $300 \mathrm{~mm}(\lambda / 4$ of $250 \mathrm{MHz}$ ) long, and are carefully assembled to make $50 \Omega$ stripline impedance. The transverse kicker shunt impedance $R_{k}$ is given by [6]

$$
R_{k} T^{2}=2 Z_{L}(2 g v / h \omega)^{2} \sin ^{2}(\omega l / v)
$$

where $Z_{L}$ is the line impedance $(50 \Omega)$ of the stripline, $g$ is the stripline coverage factor, $v$ is the beam velocity, $l$ is the length of striplines, and $h$ is the distance between striplines. It is $6.5 \mathrm{k} \Omega$ at $250 \mathrm{MHz}$ cutoff frequency for the PLS kicker.

\section{TESTS AND RESULTS}

\subsection{Tune-up}

Before turning on the feedback system, all the system components are tuned up with beam signal. Frontend and backend signal line lengths and the overall delay are adjusted within several ten picoseconds with individual line length adjusters. Phase of the local oscillator signal is also adjusted to get the maximum detection at the mixer. Using a line length adjuster and a variable attenuator, the depth and the frequency of notches are fine-tuned with the beam signal. Harmonic rejection is better than $25 \mathrm{~dB}$ in all harmonics from $\mathrm{DC}$ to $250 \mathrm{MHz}$. 


\subsection{Tests}

Since the spontaneous transverse beam instability is hardly observed in the PLS, we have driven the beam at the betatron resonance frequency using a signal generator, power amplifiers and the stripline kickers of the tune measurement system. At the resonance frequency, a coherent beam oscillation is excited with the $4 \mathrm{~mm}$ peak to peak amplitude as shown in Fig. 2. When the feedback system is turned on after all tune-ups, the beam oscillation disappears in the beam image monitor. Snapshots of the oscillating beam and damped beam are also shown in Fig. 2. In the beam spectrum, betatron sidebands are also damped around $30 \mathrm{~dB}$ in the full bandwidth of the system as shown in Fig. 3. The remaining sideband amplitude is maintained by the resonance excitation of the beam.

For the better performance of the system, we have changed pickup electrodes to 7BPM2 and 8BPM2 where the dispersion function is zero and the betatron functions are much larger than 6BPM6 and 7BPM6 $\left(\beta_{h}=11.0844\right.$ and $\left.\beta_{v}=6.711\right)$. Unfortunately, after the BPMs are changed, transverse feedback system is not working as good as before. High frequency sidebands above 150 $\mathrm{MHz}$ are not damped efficiently. It seem to be very sensitive to the change of the machine tune. Further beam study and tune-ups has to be performed for the normal operation of the PLS transverse feedback system.

\section{CONCLUSION}

A transverse feedback system is developed and is undergoing testing now in the PLS. With pickup electrodes at low betatron functions and finite dispersion, we demonstrate the $30 \mathrm{~dB}$ suppression of the betatron sidebands in DC to $250 \mathrm{MHz}$ band. When the pickup electrodes are changed to the large beta and dispersion free region, however, the feedback system does not work as good as before. Further beam studies and tuning works have to be performed for the normal operation.
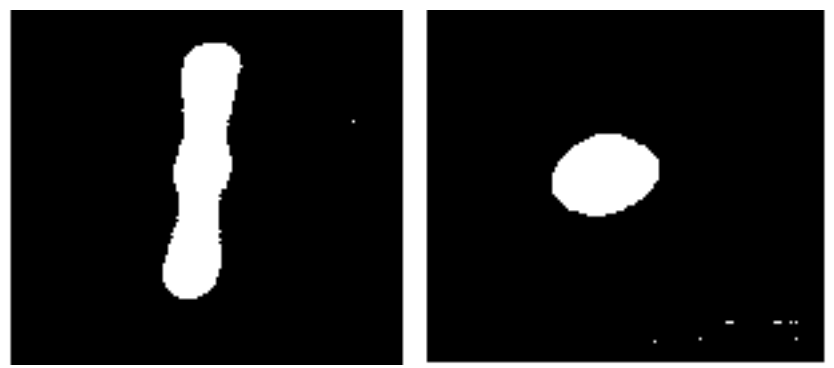

Figure 2: Beam images before and after feedback-on. Because of the automatic gain control of CCD camera, beam image after damping looks larger than before.

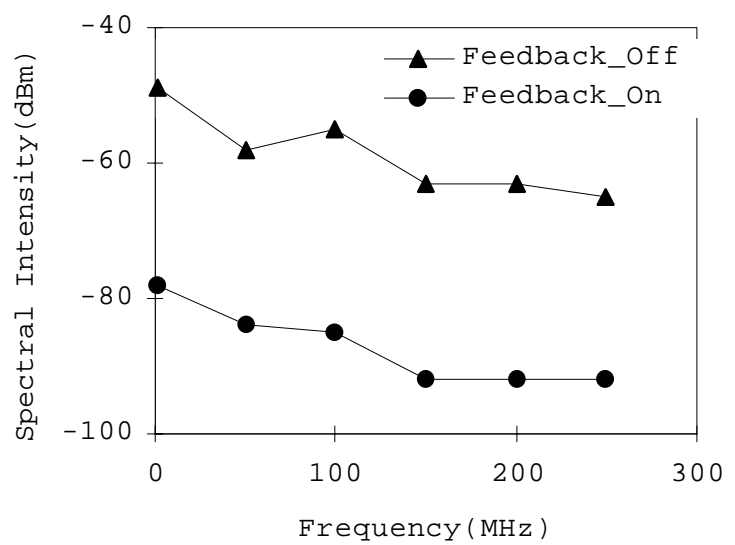

Figure 3: Betatron sidebands plotted for feedback on and feedback off.

\section{REFERENCES}

[1] M. Kwon et. al., "Instability Studies in the Pohang Light Source," Proc. of 1996 EPAC, Sitges (1997).

[2] J. Fox et. al., "Operation and Performance of a Longitudinal Damping System Using Parallel Digital Signal Processing," Proc. of 1994 EPAC, London, (1994).

[3] W. Barry et. al., "Commissioning of the ALS Transverse Coupled Bunch Feedback System," Proc. of 1995 PAC, London (1994).

[4] J. Galayda, "Performance of a Correlator Filter in Betatron Tune Measurement and Damping on the NSLS Booster," IEEE Trans. Nucl. Sci., NS-32, No.5 (1985).

[5] J. Y. Huang et. al., "Electron and Photon Beam Diagnostics in the PLS Storage Ring," AIP Conf. Proc. 333 (1995).

[6] D. A. Goldberg and G. R. Lambertson, "Dynamic Devices: A Primer on Pickups and Kickers," AIP Conf. Proc. 249 (1992). 